\title{
Research on Gait Recognition Algorithm Based on Multi-Source Information
}

\author{
Wei Xiong \\ Jiangxi University of Engineering, Xinyu, Jiangxi, 338029, China
}

Keywords: Gait recognition, Mkrvm, Multi-source informaiton

\begin{abstract}
Gait recognition technology has been well applied in many fields, and has become the representative of the second generation of biometric recognition technology, which promotes the development of pattern recognition, signal processing and other fields. The key of gait recognition is to find the proper gait features and classification methods, so as to improve the accuracy of gait recognition. In this paper, for the five gait of upstairs, downstairs, uphill, downhill and flat ground walking, we collect the multi-source information, and propose to utilize MKRVM to build gait recognition algorithm. Through the comparison of recognition accuracy results by different classifiers, it shows that MKRVM algorithm has higher recognition rate than single kernel SVM and RVM.
\end{abstract}

\section{Introduction}

Gait refers to the movement posture of the whole body in the process of movement. Gait recognition is a research topic involving pattern recognition, artificial intelligence and many other fields, because it has a wide range of application value in medical and other fields. This paper focuses on gait recognition algorithm based on multi-source information fusion. We take electromyographic (EMG) signal, knee angle signal and plantar pressure signal as gait recognition information source, and propose to utilize MKRVM algorithm to identify five gait patterns of upstairs, downstairs, uphill, downhill and flat ground walking. We choose the appropriate kernel function for each signal's characteristics, and optimize the parameters of the kernel function to get the optimal kernel function combination. Finally, we compare the recognition accuracy of different classifiers, and determine the kernel function with the highest recognition rate for each information source, so that build the optimal gait recognition algorithm.

\section{The Main Characteristic Parameters of Human Gait}

The main characteristic parameters of human gait include spatiotemporal parameters, joint angle parameters, lower limbs kinematics parameters and lower limbs muscle characteristic parameters.

\subsection{Spatiotemporal Parameters}

Spatiotemporal parameters refers to the characteristics of gait cycle, step length, stride length, walking speed, etc. Gait cycle refers to the time course from the foot landing to the foot landing again. It can be divided into supporting period and swinging period according to the position of the lower limbs when walking.

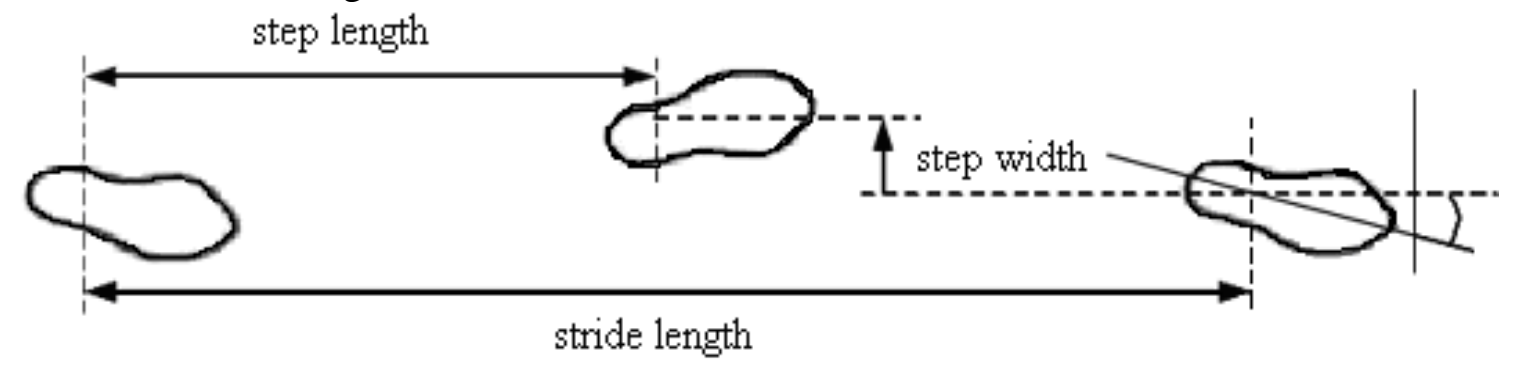

Figure 1 Step Characteristic Parameters of Gait.

The step characteristic parameters of gait is the spatial feature of foot landing, including walking 
speed, step length and stride length, as shown in Figure 1.

\subsection{Joint Angle Parameters}

Joint angle parameter refers to the motion characteristics of hip, knee and ankle joints, such as the angle, angular velocity and angular acceleration of each joint. Hip joint angle refers to the angle between the longitudinal axis of the trunk and the longitudinal axis of the femur. Knee joint angle is the angle between the extension line of the longitudinal axis of the femur and the parallel line of the longitudinal axis of the tibia. Ankle joint angle is the angle between the fifth metatarsal and the lateral midline of fibula minus $90^{\circ}$.

\subsection{Lower Limbs Kinematics Parameters}

Lower limb kinematics parameters mainly include plantar pressure, various joint moments, etc.

\subsection{Lower Limbs Muscle Characteristic Parameters}

Lower limb muscle characteristic parameters is mainly reflected in the EMG signal of the lower limb in the state of movement.

From the above descriptions of gait parameters, it can be seen that the spatiotemporal parameters are more intuitive, and the joint angle parameters and kinematic mechanics parameters can describe the gait details in more detail. In this paper, we mainly study the lower limb EMG signal, plantar pressure, hip angle, acceleration and other signals, so as to lay a foundation for further research on gait recognition technology.

\section{Collection of Multi-Source Gait Information}

From the point of view of many kinds of motion modes and gait recognition, this paper obtains the multi-source information such as the surface electromyography signal of lower limbs, the angle of hip joint, acceleration, foot pressure and so on, and taking use of multi-sensor fusion technology to realize multi-sensor complementary advantages, so that realize the recognition of more kinds of motion and behavior modes.

We selected 50 healthy subjects of different ages and genders between 20 and 50 years old as the subjects. The personal information of each subject are shown in Table 1.

Table 1 Personal Information of the Subjects.

\begin{tabular}{|c|c|c|c|c|c|}
\hline Gender & Weight $(\mathrm{kg})$ & Height $(\mathrm{cm})$ & $\begin{array}{c}\text { Leg length } \\
(\mathrm{cm})\end{array}$ & $\begin{array}{c}\text { Knee width } \\
(\mathrm{cm})\end{array}$ & $\begin{array}{c}\text { Ankle width } \\
(\mathrm{cm})\end{array}$ \\
\hline Male (25) & $62.98 \pm 14.79$ & $175.00 \pm 30.44$ & $990.20 \pm 31.92$ & $104.10 \pm 9.98$ & $74.10 \pm 6.98$ \\
\hline $\begin{array}{c}\text { Female } \\
(25)\end{array}$ & $54.01 \pm 1.97$ & $163.9 \pm 11.39$ & $910.00 \pm 47.95$ & $95.10 \pm 5.80$ & $65.00 \pm 3.96$ \\
\hline
\end{tabular}

In the multi-source information collection system, the sampling frequency of the wireless EMG signal sensor is $1000 \mathrm{~Hz}$. EMG signal is transmitted to computer via wireless network. Collect the hip joint motion signal and plantar pressure signal through the acquisition card to the computer, the sampling frequency is $1000 \mathrm{~Hz}$.

In order for the subjects to complete the five gait of upstairs, downstairs, uphill, downhill and flat ground walking, we build a standard test platform. The experimental platform consists of stairs, slopes and flat panels. The staircase is provided with 6 steps, which can complete 3 gait cycles, and the step height is $150 \mathrm{~mm}$, which is widely used; the slope is a 15 degree slope of the barrier free passage, and the number of gait cycles that can be completed is determined by the characteristics of personal walking, generally 3-4 cycles; the plane board connects stairs and slopes, more in line with walking gait in daily life. The experiment of flat ground walking can be done in the laboratory with large activity space, and it is not necessary to be confined to the flat plate.

Each subject collected 5 groups of data for each gait, then rested for 5 minutes, relaxed the leg muscles to avoid the influence of muscle fatigue on the experiment, and ensured the objectivity of the experimental data. All subjects were required to have no strenuous activity or muscle fatigue 
within 24 hours before the experiment. Before the experiment, isopropanol was used to wipe the surface and silver detection strip of the EMG signal sensor to remove the residue. After the sensor position was determined, the sensor was fixed on the cleaned skin with double-sided adhesive tape matching the silver detection strip.

The experimental contents are as follows:

a) Upstairs and downstairs experiment;

b) Uphill and downhill experiment;

c) Flat ground walking experiment;

d) The subjects walking at an asynchronous speed on the indoor treadmill.

Before the start of experiment d), the subjects were asked to warm up for 1.0-1.5 minutes at the speed to be measured on the treadmill. In order to ensure the natural posture of the subjects, after the start of the experiment, when the speed of the subjects is stable, start to collect data.

The experimental steps are as follows:

a) First of all, the subjects are asked to learn about the whole experimental process to ensure that they understand the experimental operation correctly;

b) The skin of the subjects was treated and surface EMG electrodes were pasted;

c) Before the experiment, the subjects should fully grasp the action essentials and perform 2-3 familiar operations;

d)The subjects repeated walking gait of upstairs, downstairs, uphill, downhill and different speeds, each gait repeated 5-10 times, and collected multi-source signal data;

e) After the collected data is confirmed to be valid, the experiment ends.

\section{Gait Recognition Algorithm Based on Multi-Source Information}

\subsection{Mkrvm Classification Algorithm}

Relevance vector machine (RVM) is a kind of machine learning method based on Bayesian framework theory. RVM is still a two classifier in essence. Different kernel functions correspond to the feature space and nonlinear mapping of different information sources. The mapping ability of multi kernel functions is stronger, and the performance of decision functions can be improved by using multiple kernel functions at the same time. Multiple kernel relevance vector machine (MKRVM) also adopts hierarchical Bayesian model, which can fuse different feature information through multiple likelihood functions, so as to get better combination features. The principle of MKRVM is shown in Figure 2, where $S$ represents the category of information data, $\beta_{1}, \beta_{2}, \cdots \beta_{S}$ represent the combined kernel parameter.

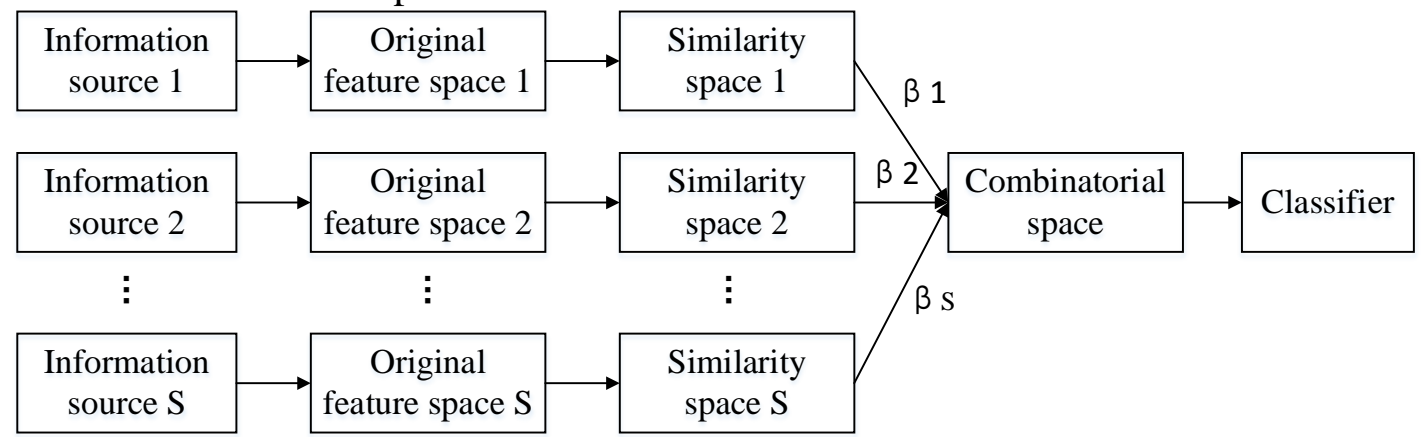

Figure 2 The Principle of Mkrvm.

Assume that there are $S$ kinds of information sources, and $S$ feature spaces after feature extraction are obtained by certain methods, then the sample data sets can be represented as $X^{S}=\left\{x_{i}, t_{i}\right\}_{i=1}^{N} . N$ is number of samples; $D$ is the dimension of eigenvector; $C$ is number of categories. When the kernel function is determined, the kernel matrix is expressed as $K^{S}$. The multi-core matrix is defined as: 


$$
K^{\beta}\left(x_{i}, x_{j}\right)=\sum_{S=1}^{S} \beta_{S} K^{S}\left(x_{i}^{S}, x_{j}^{S}\right)
$$

According to the assisted regression objective $Y \in R^{N \times C}$ and weight parameter $W \in R^{N \times C}$, then obtain the standard noise model as:

$$
y_{n c} \mid w_{c}, k_{n}^{\beta} \sim N_{y n c}\left(k_{n}^{\beta} w_{c}, 1\right)
$$

Introduce multiple probability connection function $t_{n}=i, y_{n i}>y_{n j}$, thus we can get that the multiple probability likelihood function as:

$$
P\left(t_{n}=i \mid W, k_{n}^{\beta}\right)=\varepsilon_{p(u)}\left\{\prod_{j \neq 1} \phi\left(u+k_{n}^{\beta}\left(w_{i}-w_{j}\right)\right)\right\}
$$

The regression factor $w_{n c}$ in parameter $W$ obeys to the standard normal distribution with mean value of 0 and variance of $a_{n c}{ }^{-1}$. Scale matrix $A \in R^{N \times C}$ consists of $a_{n c}$, which obeys the Gamma distribution of super parameters $\tau$ and $v$.

\subsection{Kernel Function Selection and Algorithm Flow}

Kernel function selection is the key to the accuracy of MKRVM recognition. The distribution of different data information in the feature space is different, so choosing the appropriate kernel function for each information data will have a corresponding impact on the final classification.

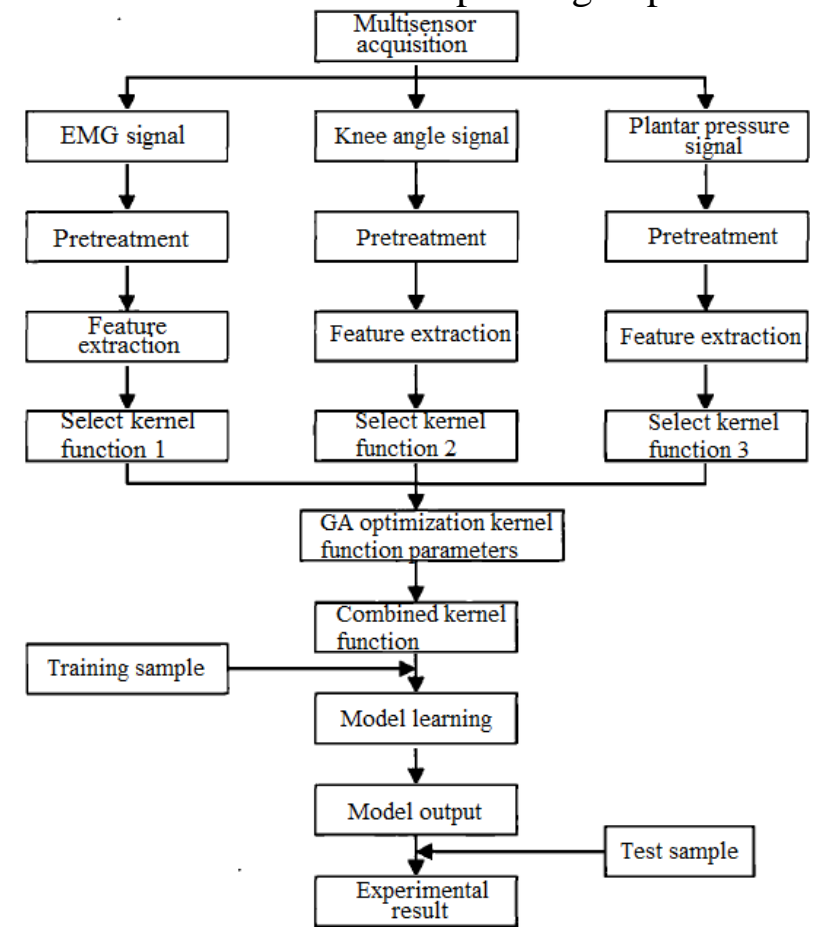

Figure 3 The Gait Recognition Algorithm Flow.

One of the main advantages of SVM and RVM classifier algorithm is the use of kernel function. The common kernel functions are linear kernel, polynomial kernel, radial basis kernel and sigmoid kernel. Their formulas are as follow:

$$
\begin{array}{r}
K(u, v)=u^{T} v \\
K(u, v)=\left(g \cdot u^{T} \cdot v+c\right)^{d} \\
K(u, v)=\exp \left(-g \cdot|u-v|^{2}\right)
\end{array}
$$




$$
K(u, v)=\tanh \left(g \cdot u^{T} \cdot v+c\right)
$$

In the formulas, $g$ is the coefficient of vector product in kernel function; c is the independent term of kernel function; $d$ is the width of polynomial kernel function.

In this paper, the gait recognition method of lower limbs based on MKRVM classification algorithm integrates gait information with different characteristics. The gait recognition algorithm flow is shown in Figure 3.

\section{Experimental Results and Analysis}

MKRVM classification outputs the probability of various gait, and the gait corresponding to the maximum probability value is the recognition result. This paper analyzes the experimental data of five different gait. Then, the kernel function performance of different gait features is analyzed by comparing single kernel SVM with single kernel RVM. We randomly divide the offline sample set into three parts: $70 \%$ of which are used for training model, $20 \%$ for cross validation parameter optimization, and the remaining $10 \%$ for offline test. In the process of parameter optimization, 20 samples are randomly selected from the cross validation samples each time, and the accuracy of cross validation is calculated. When the accuracy rate reaches a predetermined value (90\%), it terminates, and the maximum generation number is set to 500 generations.

Taking the experimental data of flat ground walking as example, the single core classifier recognition results with SVM and RVM algorithm is shown in Table 2

Table 2 Single Kernel Classifier Recognition Results with Svm and Rvm Algorithm.

\begin{tabular}{|c|c|c|c|c|c|c|}
\hline \multirow{2}{*}{$\begin{array}{c}\text { Kernel } \\
\text { function }\end{array}$} & \multicolumn{2}{|c|}{ EMG signal / \% } & \multicolumn{2}{c|}{ Knee angle signal / \% } & \multicolumn{2}{c|}{ Plantar pressure signal / \% } \\
\cline { 2 - 7 } & SVM & RVM & SVM & RVM & SVM & RVN \\
\hline Linear & $81.18 \pm 0.21$ & $81.49 \pm 0.22$ & $84.31 \pm 0.58$ & $82.41 \pm 1.61$ & $79.44 \pm 1.63$ & $80.13 \pm 0.24$ \\
\hline Polynomial & $76.27 \pm 0.65$ & $83.25 \pm 0.79$ & $78.52 \pm 0.31$ & $76.45 \pm 0.90$ & $78.23 \pm 0.16$ & $77.36 \pm 0.21$ \\
\hline Radial basis & $89.22 \pm 1.02$ & $90.63 \pm 0.52$ & $90.33 \pm 0.78$ & $88.36 \pm 0.15$ & $82.16 \pm 0.33$ & $78.75 \pm 0.68$ \\
\hline Sigmoid & $84.34 \pm 0.48$ & $85.97 \pm 0.86$ & $90.04 \pm 0.57$ & $91.45 \pm 0.45$ & $80.12 \pm 0.15$ & $80.09 \pm 1.04$ \\
\hline
\end{tabular}

For the parameter setting of MKRVM, this paper uses GA to optimize the parameters of kernel function of MKRVM. For the five gait of upstairs, downstairs, uphill, downhill and flat ground walking, the recognition results of different classifiers are shown in Table 3.

Table 3 Recognition Results Of Different Classifiers.

\begin{tabular}{|c|c|c|c|c|}
\hline \multirow{2}{*}{ Gait pattern } & \multicolumn{4}{|c|}{ Recognition accuracy rate / \% } \\
\cline { 2 - 5 } & BP & SVM & RVM & MKRVM \\
\hline Upstairs & $85.56 \pm 1.52$ & $87.20 \pm 0.59$ & $85.08 \pm 0.54$ & $93.13 \pm 0.60$ \\
\hline Downstairs & $86.19 \pm 0.85$ & $86.72 \pm 0.77$ & $88.35 \pm 1.29$ & $92.46 \pm 0.38$ \\
\hline Uphill & $84.32 \pm 0.78$ & $85.06 \pm 0.59$ & $84.46 \pm 1.06$ & $85.97 \pm 0.72$ \\
\hline Downhill & $86.16 \pm 0.43$ & $85.97 \pm 0.88$ & $84.36 \pm 0.25$ & $87.26 \pm 0.78$ \\
\hline Flat ground walking & $84.48 \pm 1.22$ & $88.14 \pm 0.45$ & $89.36 \pm 0.80$ & $95.55 \pm 1.01$ \\
\hline
\end{tabular}

It can be seen that the recognition accuracy of MKRVM is higher than that of single kernel SVM and single kernel RVM. Through the analysis of experimental results, MKRVM combines the gait information of multi-source signals, which is better than SVM and RVM. It can be seen that the performance of using the combination of multiple kernel functions is better than using a single kernel function.

\section{Conclusion}

In this paper, the surface EMG signal, plantar pressure signal and inertia signal of five gait of upstairs, downstairs, uphill, downhill and flat ground walking, are collected by multi-source information acquisition system, and gait recognition is carried out by multi-core MKRVM. Through the comparison of recognition accuracy results by different classifiers, it shows that MKRVM 
algorithm combines multi-source information such as EMG signal, plantar pressure signal and inertia signal, selects the best kernel function and parameters for each information source, and has higher recognition rate than single kernel SVM and RVM.

\section{Acknowledgment}

Supported by Science and Technology Research Project of Education Department of Jiangxi Province 2019: Research on gait recognition Algorithm based on multi-source information (GJJ191195).

\section{References}

[1] Zheng, Shuai, Zhang, Junge, Huang, Kaiqi,et al. Robust view transformation model for gait recognition[J]. Research Center for intelligent perception and Computing, 2011, 263(4):2073-2076.

[2] Chen C, Liang J, Zhao $\mathrm{H}$, et al. Frame difference energy image for gait recognition with incomplete silhouettes [J]. Pattern recognition letters, 2009, 30(11):977-984.

[3] Lai Z, Xu Y, Member, et al. Human Gait Recognition via Sparse Discriminant Projection Learning [J]. IEEE Transactions on Circuits \& Systems for Video Technology, 2014, 24(10):16511662.

[4] Ning J, Victor S, Chang-Tsun L. On view-invariant gait recognition: a feature selection solution [J]. Iet Biometrics, 2018, 7(4):287-295.

[5] Tafazzoli F, Bebis G, Louis S, et al. Genetic feature selection for gait recognition [J]. Journal of electronic imaging, 2015, 24(1):013036.1-013036.14.

[6] Chattopadhyay P, Roy A, Sural S, et al. Pose Depth Volume extraction from RGB-D streams for frontal gait recognition [J]. Journal of Visual Communication \& Image Representation, 2014, 25(1):53-63.

[7] Yiwei H, Junping Z, Hongming S, et al. Multi-task GANs for View-Specific Feature Learning in Gait Recognition [J]. IEEE Transactions on Information Forensics \& Security, 2018:1-1. 